\title{
MIRANDO HACIA EL FUTURO CON PENSAMIENTO COMPLEJO EN LA EDUCACION SUPERIOR.
}

Paredes Cabezas Maribel ${ }^{1}$, Zoila López Miller ${ }^{2}$ \& Velasteguí López Efraín ${ }^{3}$

\begin{abstract}
The rapid changes that are being operated in the different scenarios of science and technology in a globalized world, demand for this third millennium, the pose and practice of a new educational paradigm and challenges him to Edgar Morin fractionation of knowledge and segmentation of knowledge, and supports a systemic position from the perspective of complex thinking with the view that nothing in the world is separate, all are in relation and interaction, humans, objects, nature, medium, develop as a whole, they are needed. The philosopher Edgar Morin opposes the isolation of the objects of knowledge, rehabilitates its context, reinserted into the totality to which they belong. The complex thought process plays a thoughtful and multidimensional exploration of the facts from the uncertainty and education is ripe for discernment space. The theme addresses the participatory demand of teachers and students of the university to open the enclosure of traditional teaching and learning towards a dialogic academia to ensure constructive criticism and professional training, engaging with the community, the environment in which lives and encompasses the theoretical underpinnings of complex thought.
\end{abstract}

\section{Keywords:}

Paradigm, systemic, interaction, knowledge, multidimensional, dialogical thinking.

\section{CÓDIGO UNESCO: Educación 531204}

\section{RESUMEN}

Los cambios vertiginosos que van operándose en los distintos escenarios de la ciencia y la tecnología en un mundo globalizado, exigen para este tercer milenio, el planteamiento y la práctica de un nuevo paradigma educativo, así interpretando a Edgar Morín quien ante el fraccionamiento del conocimiento y la segmentación de los saberes, sustenta una posición sistémica desde la perspectiva del pensamiento complejo con el criterio de que ningún elemento del mundo se encuentra separado, todos se encuentran en relación e interacción, los humanos, los objetos, la naturaleza, el medio, se desarrollan como un todo, es decir se necesitan entre sí. Es importante también señalar que Edgar Morín se opone al aislamiento de los objetos del conocimiento, los rehabilita a su contexto, los reinserta en la globalidad a la que pertenecen. Razón por la cual el pensamiento complejo asume un proceso de exploración reflexiva y multidimensional de los hechos, a partir de la incertidumbre, convirtiendo a la educación en el espacio propicio para el discernimiento. Mirar hacia el futuro implica abordar la exigencia participativa de docentes y estudiantes de la universidad, a fin de abrir el encierre de la enseñanza-aprendizaje tradicional

${ }^{1}$ Facultad de Contabilidad y Auditoría, Universidad Técnica de Ambato, Ambato, Ecuador, m.paredesi@uta.edu.ec

2 Facultad de Ciencias Administrativas, Universidad Técnica de Ambato, Ambato, Ecuador, z.lopezl@uta.edu.ec

${ }^{3}$ Facultad de Ciencias Administrativas, Universidad Técnica de Ambato, Ambato, Ecuador le.velastegui@uta.edu.ec 
hacia una academia dialógica que garantice la formación crítica y constructiva del profesional, buscando una implicación y participación directa con la colectividad y el medio en el que habita, para ello se ha tomado como guía los sustentos teóricos del pensamiento complejo.

\section{Palabras clave:}

Paradigma, sistémico, interacción, conocimiento, pensamiento multidimensional y dialógico.

\section{INTRODUCCIÓN}

La globalización, la internacionalización, el avance tecnológico y la educación, constituyen pilares fundamentales de transformación y desarrollo, en este contexto la educación juega un papel fundamental para generar el cambio, partiendo para ello de la noción integral del hombre, del mundo, del conocimiento, del aprendizaje, del entorno, con pleno sentido unificador, desarrollando la independencia hacia el paradigma de lo autónomo, lo constructivo y lo revelador. Debe hablarse de una mente humanística, donde se le otorgue al ser, la supremacía para decidir por sí mismo, con principios y conocimientos no separados; sino con un motivo cooperativo, integrador, compartido, dialógico, intercomunicado y dialéctico.

En este aporte se trazan enfoques esenciales para el debate de la educación superior en base al pensamiento de Morín, plasmado en una de sus obras más importantes denominada "Los siete saberes necesarios para la educación del futuro" la misma que contribuyen ampliamente en esta nueva concepción. Morín abre estos espacios para la transformación del pensamiento, el cual en el aprendizaje juega una función específica y promulga la apropiación y difusión de siete saberes que la educación del futuro debería tratar en forma integral y en toda cultura. El pensamiento de Edgar Morín plantea una transformación para la educación superior basada en una auténtica asociación entre disciplinas científicas, integrando las capacidades: crítica, autocrítica y reflexiva en la construcción del conocimiento, avizora una visión holística y de interrelación con todos los elementos y esferas del conocimiento, así como con la interacción del ser humano tratando de agrupar la complejidad del contexto y del mundo.

Morín motiva el cambio académico reformulando las políticas, planes y programas, con un nuevo paradigma, acogiendo y desarrollando el pensamiento complejo, con lo que se espera un aseguramiento de calidad académica y generación constante de conocimiento. Así es como la Universidad debe comprometerse y reintegrar fuerzas en la formación y proceso del pensamiento, apartando lo simplista, la ausencia de la interpretación de la complejidad, y poniendo en pie el crecimiento universal del conocimiento, ignorarlo sería un retroceso y la academia no tendría sentido.

"La complejidad de pensamiento debe ser entendida como: una red de constituyentes heterogéneas unidos de forma inseparable", que asocien "la paradoja de lo simple y lo complejo, uno y múltiple, es una secuencia interrelacionada de eventos, acciones, interacciones retroacciones, determinaciones y azares, que son componentes del mundo" en un entorno natural, pensamiento de Morín entre 1976 y 1988. 
Hoy la educación constituye un instrumento poderoso para agilizar el cambio. El pensamiento complejo reclama una educación que de valor a la afectividad y acoja el error como oportunidad para aprender, que entienda la idea de que cualquier sustancia del mundo no es un objeto solitario, sino que es inseparable de un sistema que lo sostiene como parte de su interacción, tanto de su sistema como de un todo.

En el libro, "El Metro", escrito por Morín, manifiesta que: "Un sistema es una interrelación de elementos que constituyen una entidad global o una unidad global. Tal definición comporta dos caracteres principales: el primero es la interrelación de los elementos y el segundo es la unidad global constituida por estos elementos en interacción (...) Se puede concebir el sistema como unidad global organizada de interrelaciones entre elementos, acciones o individuos" (pp.123-124).

Es pretensión de este artículo participar del debate sobre la educación superior sostenido en el pensamiento complejo, principios, teorías y supuestos que el Filósofo Edgar Morín los respalda.

\section{UNA PROXIMIDAD AL CONOCIMIENTO}

Según (Bilancio, 2006), (Costa, 2008), (González, 2012), (Laclette, Zuñiga, \& Puga Espinoza, 2010), (Rubio Matilla, 2011) "Un acercamiento al conocimiento inicia con la vida al momento de expresar palabras y acciones propias del ser humano, pudiendo generar actitudes reflexivas o no reflexivas de un líder que puede tener un aprendizaje constructivista que lleve al conocimiento previo a partir de una preparación académica por medio de base de datos y revistas académicas"

El conocimiento es una construcción ideológica del hombre fundamentado en el entendimiento y la razón, con un desarrollo constante que se remonta al momento mismo en que el hombre comenzó a experimentar, indagar, cuestionar y razonar sobre la gran cantidad de información que iba adquiriendo en su proceso evolutivo. De hecho el conocimiento llega a nosotros como un proceso y no como un acto único donde se pasa de una vez de la ignorancia a la verdad, en este sentido, el conocimiento científico es uno de los modos posibles de apropiación y generación de conocimiento, tal vez el más desarrollado para responder nuestras interrogantes a partir del estudio de hechos verificables.

El conocimiento emerge en el contexto de los movimientos elementales entre quien conoce un objeto del conocimiento, el acercamiento y el alejamiento. Estos dos actos resumen en su sencillez la esencia misma del acto de conocer

Los seres humanos han acumulado saberes sobre el entorno en que viven a partir de aumentar sus conocimientos teóricos y prácticos para transformar la realidad circundante. Este conjunto de conocimientos, así como la actividad humana destinada a conseguirlos, es lo que se denomina como ciencia. Sin embargo el concepto de ciencia no se puede aplicar a cualquier tipo de conocimiento, sino únicamente a los saberes que han sido obtenidos mediante una metodología, tal como sucede aplicando el método científico cumpliendo además con determinadas condiciones. 
El conocimiento puede ser entendido como el saber consciente y fundamentado que somos capaces de comunicar y discutir, se distingue así del conocimiento vulgar que es simplemente recordado y que no podemos someter a crítica. En la actualidad se considera que el conocimiento es un proceso, anteriormente la filosofía tradicional lo concebía como algo estático. Por ello, lo que caracteriza a la ciencia actual no es la pretensión de alcanzar un saber verdadero sino, la obtención de un saber riguroso y verificable.

Se sabe que con el pasar de los años y el avance de la tecnología es mucho más factible tener un acercamiento al conocimiento, aunque también se habla de que el acercamiento al conocimiento se inicia desde el mismo comienzo de la vida cuando aprendemos a analizar y expresar palabras.

Los líderes han sido fundamentales para llevar a su sociedad al aprendizaje por medio de reflexiones, pues gracias al conocimiento se han realizado estudios importantes como el que realizó Charles Darwin con su teoría el origen de las especies que fue debido al conocimiento adquirido por medio de las vivencias experimentadas en el trayecto de su carrera científica al visitar y conocer las Galápagos.

En este contexto, podemos entonces entender que para lograr una aproximación efectiva de la generación y difusión del conocimiento, resulta importantísimo dominar sobre ciencia y tecnología, pues gracias al empleo de grandes herramientas se han podido generar libros, revistas académicas que ayudan a activar el conocimiento previo de algo. Se dice que para llegar a un buen nivel de conocimiento el ser humano debe estar en constante aprendizaje.

También el acercamiento al conocimiento puede surgir a partir de los problemas pues, el ser humano al enfrentarse a una situación problémica debe analizar la misma y aprender de los errores. El conocimiento es la base para cualquier estudio, pues hay que tener un acercamiento sobre algún tema para poder desarrollarlo mediante investigaciones, siempre es importante saber qué es lo que se está haciendo.

"El conocimiento es un saber fundado, crítico, conjetural, sistematizado y metódico sobre aspectos del universo". (Laura.Argentina.) Todo conocimiento es una relación en el que están interactúan dos elementos, el sujeto que es cognoscente y el objeto que es conocido. "El conocimiento científico es un pensamiento dinámico en la conciencia de los sujetos capaz de utilizar la reflexión crítica sobre un problema. (...) Se requieren de ciertos pasos para llegar a él sin embargo es necesario un pensamiento categorial para acceder, por lo cual exige la categoría de "totalidad" entendida como la apertura a la realidad que vivimos para concretar en un tópico específico”. (Carlos Rodríguez)

"Es un sistema de conceptos acerca de los fenómenos y leyes del mundo externos los cuales pueden ser comprobados mediante la racionalidad y la sistematización”. (Carlos Alberto López Mtz. (Veracruz, Mex.))

"Estas respuestas" no son aisladas, "están relacionadas, la relación de un conocimiento no puede estudiarse dejando de lado al sujeto y al objeto. Un naturalista afirma que lo que la 
ciencia obtiene no son las cosas mismas sino las relaciones que existen entre las cosas. Fuera de estas relaciones no hay una realidad que conocer. Emilio Morselli (...) afirma que lo único que el hombre conoce son las relaciones que se dan entre él y las cosas"

Ante la expectación cognitiva de investigar sobre el conocimiento, hay que indicar que no existe límite, las barreras, el alcance, están condicionados a la existencia humana, vale recalcar la inferencia del paradigma Moríniano respecto al criterio de incertidumbre: el saber es un conocimiento inconcluso, al respecto Roger Ciurana (1997, p. 298), señala que pensar la complejidad " es un intento de establecer un diálogo distinto con lo real lo menos rutilante posible, en este sentido, se trata de un pensamiento menos desmesurado y menos híbrido que el pensamiento reductor y disyuntor".

Cuando se pide al pensamiento que explique las incertidumbres, las leyes, los contenidos, que ponga templado la realidad, que reflexione y relacione el todo con sus partes, que revele la verdad de aquello que rige tales conocimientos, ahí se está poniendo en juego un pensamiento complejo.

En el conocimiento desde el pensamiento complejo, el método se construye en la medida que avanza lo investigativo. "El método es una búsqueda que se inventa y se reconstruye continuamente (...) es una actividad pensante del sujeto viviente, no abstracto; un sujeto capaz de aprender, inventar, crear en y durante el caminar" (Morín, Roger Ciurana y Motta, 2002. pp. 15-16). Desde la óptica de la complejidad no se persigue sólo explicar, sino también comprender; de la misma manera, las explicaciones no pueden ser únicamente lineales, sino que deben tener sentido de lo multidimensional, de lo lógico y lo dialógico. Por ello, Morín (1988 p.20) define el conocimiento como "un fenómeno multidimensional en el sentido de que, de manera inseparable, a la vez, es físico, biológico, cerebral, mental, psicológico, cultural y social".

\section{MORÍN Y EL CONOCIMIENTO}

Morín combate la visión del mundo producto de la racionalidad científica tradicional, pero no niega que el conocimiento humano (reflejado en la ciencia) haya generado cambios más o menos positivos en las sociedades. Morín insiste, sobre todo, que estos conocimientos han llegado, a lo largo de los siglos, al extremo de borrar y desconocer la naturaleza mismo de aquello que estudia: en el marco de las especializaciones disciplinarias en el transcurso del siglo XX (especializaciones que originan desde el inicio de la modernidad), se ha perdido la perspectiva de complejidad, multiplicidad y prolijidad de los factores que intervienen en la construcción de cada elemento en el universo(ya sea que hablemos de "elementos" sociales, físicos, biológicos u otros), así como su vínculo con las diferentes "partes" de los sistemas a los que pertenecen.

Estos progresos científicos han sido dispersos y aislados a causa de, precisamente, la especialización absoluta de dicho conocimiento y a su afán, dada esa peculiaridad de evitar el contexto y las globalidades como aspectos esenciales de su análisis. Así las realidades globales (complejas) se han quebrado y lo humano se ha desarticulado: sus múltiples dimensiones (biológica, social, cultural, económica, física, histórica, etc.) se han esparcido en una serie de saberes que muy pocas veces tienen interrelación entre sí. Este ha sido, según Morín, el legado de la modernidad y de los enfoques utilitaristas del conocimiento 
que, desde Descartes, Bacon y hasta, Marxhan han predominado en el ambiente científico y académico.

Este no es un problema menor, sino que posee la mayor trascendencia, pues de esta tradición centenaria (y su visión simplificada del mundo) devienen, según Morín, los principales problemas que actualmente, intenta combatir el denominado "pensamiento de la complejidad". Según esta perspectiva, resulta evidente que, bajo las condiciones impuestas por el conocimiento fragmentario, las personas han perdido su capacidad para contextualizar los saberes, es decir, su capacidad para integrarlos a los conjuntos (o sistemas) a los que pertenecen (Morín 1999.p2) y con ello han perdido la verdadera noción de lo que representa, la condición humana, la cual para Morín, es una noción que contempla lo humano en tanto que "es a la vez físico, biológico, psíquico, cultural, social e histórico, es decir una identidad compleja y común a todos los demás humanos"(Morín 1999.p.3. En este sentido, surge, la necesidad de desarrollar la aptitud de las personas para ubicar el conocimiento y la información dentro de un contexto y dentro de un conjunto.

\section{LA COMUNICACIÓN EN LA EDUCACIÓN}

Según los autores:(Olivos, 2005); (TISCAR, 2006); (JIMÉNEZ, s.f); (Castillo, 1998); (Kaplúm, 2003). "Consideran que la comunicación no puede estar separada de la educación dentro de una sociedad donde la escuela ya no es como se creía tradicionalmente para esto debe considerarse la retroalimentación y el rol de sus actores con el fin de establecer relaciones entre maestros y alumnos, maestros y colegas, maestros y padres."

"No hay Comunicación sin Educación, como no hay Educación sin Comunicación" (TISCAR, 2006). Para referirnos a la comunicación en la educación debemos tener en cuenta que se tratan de términos distintos y en la actualidad es necesario fusionar la comunicación con la educación, aunque la mayoría de las veces se ha creído que modernizar el sistema educativo se trata de ajustarse a los nuevos avances tecnológicos, considerándolo como la solución a los problemas pero no a fondo, claro está que dichos avances incluso reemplazan al profesor físicamente por una imagen en el ordenador, sin embargo esto le quita lugar al tema de la comunicación ya que se trata del condimento necesario con lo cual se puede dialogar, participar, distinguir y reflexionar de manera colectiva.

La comunicación en la educación es lo que debe implementarse, y abandonar las viejas perspectivas que el profesor solo tenía la obligación de dictar su materia y marcharse, las cosas han cambiado ahora se trata de generar una convivencia entre el profesor- alumno y entre compañeros construyendo así los cuatro pilares de la educación: Aprender: Conocer, hacer, convivir, y ser.

La educación y la comunicación, tienen como principal meta lograr ciudadanos responsables y participativos, con capacidad crítica, creadores en común de soluciones de los problemas, que cuestionen la información que reciben, que informen, opinen, se procuren sus propias fuentes de información y que las comparen con la que genera el poder mediático. 
Las nuevas tecnologías agilizan los procesos informativos, pero no son la solución si no promueven la comunicación pero eso no hay que interpretarlo como una pésima herramienta.

Está comprobado que los estudiantes aprenden más cuando hay momentos dinámicos como chistes, música, etc. El profesor debe buscar distintas técnicas de aprendizaje para compartir los conocimientos necesarios pero sin omitir la comunicación, obviamente no se puede dejar apartado la tecnología, el éxito está en fusionar las Ntic's, comunicación, y educación, componentes tan simples pero que ciertas veces al docente se le olvida.

\section{PENSAMIENTO COMPLEJO}

(Caballero, 2008) "Concreta que el pensamiento complejo se designa como una comprensión del mundo, como entidad donde todo se encuentra entrelazado, como en un tejido compuesto de finos hilos. También se puede señalar, siguiendo su idea el pensamiento complejo es ante todo un pensamiento que relaciona." El pensamiento complejo es una compilación de ensayos y presentaciones realizadas entre 1976 y 1988, los años durante los cuales el método comienza a cobrar forma como estructura articulada de conceptos.

El estudio de cualquier aspecto de la experiencia humana ha de ser por necesidad, en que vemos cada vez más que la mente humana, sin bien no existe sin cerebro, tampoco existe sin tradiciones familiares, sociales, genéricas, étnicas, raciales, que solo hay mentes encarnadas en cuerpos y culturas y que el mundo físico es siempre el mundo entendido por seres vivos y culturales.

Según (Solana, 2005) "La noción de pensamiento complejo fue acuñada por el filósofo francés Edgar Morín y refiere a la capacidad de interconectar distintas dimensiones de lo real. Ante la emergencia de hechos u objetos multidimensionales, interactivos y con componentes aleatorios o azarosos el sujeto se ve obligado a desarrollar una estrategia de pensamiento que no sea reductiva ni totalizante, sino reflexiva, Morín denominó a dicha capacidad como pensamiento complejo.”. Edgar Morín ve a los procesos sociales en movimiento incluye preocupaciones éticas como centrales para la observación de procesos sociales, entendiendo que la ética también evoluciona en sí misma, como un proceso social.

(Grinberg, 2002) “Concreta que el Pensamiento Complejo de Morín, se reconoce como un pensamiento que relaciona y complementa su objeto y sujeto de estudio en el todo, a través de sus efectos, defectos, dinamismo y estática, reconociendo la interrelación del todo con sus partes y viceversa, dentro de un entramado." Es nuestra responsabilidad y nuestro destino el que está en juego, el pensamiento complejo es una aventura, pero también un desafío.

La necesidad de un pensamiento complejo, afirma Morín, se impondrá en tanto vayan apareciendo los límites, las insuficiencias y las carencias de un pensamiento cambiante y en esa medida estar a la altura de su desafío. Se hace necesario crear un método que, de alguna manera vaya a pensar, un pensamiento que dialogue con lo real. En Morín la complejidad no es una reducción o deslinde de la simplicidad. Al contrario, el pensamiento complejo íntegra las formas en que se aumente la forma de pensar. El pensamiento complejo se 
concibe como un pensamiento total, completo, multidimensional lo define Morín, pero se reconoce en un principio de incertidumbre. Se reconoce como pensamiento no parcelado dividido, no reduccionista, pero reconoce lo inacabado e incompleto del pensamiento.

La crítica que Morín hace del pensamiento que va aumentando lo problematiza porque considera que este pensamiento no concibe la conjunción de lo uno y lo múltiple, unifica en abstracto y anula la diversidad y por este camino se llega a la inteligencia ciega. Sin embargo, considera que la simplificación es necesaria, pero debe ser relativizada. Morín define la complejidad como un tejido de eventos, acciones, interacciones, retroacciones, determinaciones, azares que conforman el mundo de lo fenoménico, y sus rasgos son los de ordenar lo confuso, el desorden, la ambigüedad y la incertidumbre, estrategias para lograr la inteligibilidad.

En Morín la complejidad es un fenómeno cuantitativo, una cantidad de interacciones e interferencias entre un número de unidades y también determinaciones, incertidumbres, y fenómenos aleatorios. La complejidad es la incertidumbre en los sistemas organizados, esto es, que la complejidad está permeada por una mezcla de orden y desorden y en esa dirección está relacionado con el azar.

Ahora bien, Morín tiene un ejemplo para explicarnos el fenómeno simplificador. Es sobre el hombre. El hombre, afirma Morín, es un ser biológico, pero también es un ser cultural, en tanto vive inmerso en un universo de lenguaje, ideas y conciencia. El paradigma del pensamiento simplificador nos lleva a desunir el estudio del hombre, a hacerlo menos complejo, estudiándolo por partes, es decir, biológico, anatómico, psicológico, culturalmente, por separado, olvidando que el hombre es una totalidad que no existe lo uno sin lo otro. En Morín hay tres principios que nos ayuda a pensar la complejidad.

El pensamiento complejo íntegra la incertidumbre y concibe la organización que contextualiza, globaliza, pero también reconoce lo singular y lo concreto. La complejidad requiere que rompamos con nuestros tradicionales esquemas mentales en reforma del pensamiento que no nos ha habituado a concebir la dialógica de lo simple y lo complejo, de lo separable y no separable, del orden y del desorden. El paradigma del pensamiento complejo, reitera Morín, tendrá su origen en los nuevos conceptos, visiones, descubrimientos y reflexiones que puedan reunirse en una tarea cultural histórica y en ese sentido allí está su desafío.

Un punto intermedio entre el pensamiento reductor, que no observa más que los elementos y el pensamiento global, que no observa más que la totalidad, es decir, que se haya en la relación del simple y lo complejo. El método tradicional separaba para conocer y el pensamiento complejo contextualiza, globaliza y relaciona lo que está separado. En ese orden, se hace necesario aprender a ver, a concebir, a pensar y a actuar.

Una nueva ética nos propone Morín en la estrategia de su pensamiento de la complejidad y en ese sentido es pertinente para nuestra realidad latinoamericana en tanto que es originario de una nueva mirada o perspectiva sobre nuestra realidad social, política, cultural, económica desde su singularidad y su totalidad que nos conduce necesariamente al campo 
de la complejidad. Morín considera que estamos en la crueldad de las ideas, en la prehistoria del espíritu humano y solo el pensamiento complejo podrá civilizar nuestro conocimiento. Según (Najmanovich, 2008) "En el siglo XX ningún concepto se resignificó con tanta profundidad como complejo. De un uso común y científico que había perdido sus raíces y lo relacionaba con lo complicado, lo enmarañado y lo difícil de entender, retomó su sentido originario y pasó a significar una nueva perspectiva para designar al ser humano, a la naturaleza, y a nuestras relaciones con ella."

\section{PENSAMIENTO COMPLEJO Y EDUCACIÓN}

Según la bibliografía consultada; Lipman (2014); Tobón, Sánchez, Díaz \& Fraile (2006); Ameloza, Pérez, Guzmán \& Santana (2014); Carrasco, Ángel \& Garcia (2001) Navarro \& Aguilar (2013) "Los pensamientos de orden superior tienden a la complejidad, rechaza la aceptación soluciones simplistas, es una epistemología para pensar cualquier realidad de un modo diferente como se ha puesto en las ciencias forma de pensar y explicar la vida. La formación en el pensamiento complejo en la educación podrá ser una oportunidad para desarrollar una actitud crítica."

Lipman (2014) Quien manifiesta que: El pensamiento de orden superior tienden a la complejidad, rechaza la aceptación de formulaciones o soluciones simplistas, en cambio el camino no pasa por la enseñanza de destrezas para la mejora del pensamiento superficiales pincelados con un barniz lógico. (Pg.18). Tobón, Sánchez, Díaz \& Fraile (2006) manifiestan que: El pensamiento complejo es una epistemología para pensar cualquier realidad de un modo diferente como se ha puesto en las ciencias, disciplinas y epistemologías tradicionales. (pg.7). Carrasco, Angel \& Garcia (2001) mencionaron que El pensamiento constituye una norma de implicación de lo uno y lo otro, respecto a educación de la mente y el conocimiento de las disciplinas. (pg.409).

Navarro \& Aguilar (2013) mencionó: La formación en el pensamiento complejo en la educación podrá ser una oportunidad para desarrollar una actitud crítica frente a una que desde MMC se construyen a diario. (pg.115) lo complejo hace referencia a algo posible pero con sus barreras que el estudiante mismo se traza a lo largo de su educación, sabiendo que uno de los aspectos muy importantes y fundamentales del ser humano es el pensamiento, que es la base esencial para la correcta toma de definiciones no solo personales sino más bien en cualquier proyecto que se vaya a emprender a los largo del tiempo, menciono este fragmento ya que usualmente el ser humano se tiende a la complejidad es decir que en ocasiones es difícil de entender; con un enfoque objetivo relacionado al ser humano a la naturaleza y a nuestras relaciones con ello, todo se encuentra entrelazado como un tejido, es decir el pensamiento complejo es un pensamiento que relaciona y divide el campo de los conocimientos en disciplinas clasificadas, es el estudio de un todo a través de sus defectos, efectos, dinamismo y estática reconociendo la interrelación y de todos sus componentes y viceversa.

Es importante mencionar que se debería enseñar directa e inmediatamente para el pensamiento de orden superior que tiende a ser altamente significativa para los estudiantes, así como para los profesores. Con esto lograríamos que los estudiantes hagan filosofía y lograr ser un ejemplo de cómo puede estimular a su pensamiento. 
Ameloza, Pérez, Guzmán \& Santana mencionaron que: Como forma de pensar y explicar la vida, es una de las más recientes propuestas filosóficas y epistemológicas del ámbito social y científico que aportan nuevas formas de reflexionar e investigar. (pg.5) por lo que es importante asociar que el pensamiento posee recursos como la metacognición y todas aquellos tipos de modalidades que conllevan a la reflexión sobre el método que se está utilizando y el contenido de que se trata, considero que es un tipo de situación y aspecto muy indispensable en la educación ya que un estudiante para poder sobrellevar sus estudios debe realizar investigaciones las que a su vez unas serán más complejas que, por lo que debemos tomar en cuenta que se creará un pensamiento complejo ante este tipo de consulta que el alumno realice, sin embargo hay que destacar que es posible realizarlo, y esto serviría como ejemplo para que no se sigan creando paradigmas de que el pensamiento complejo en la educación no afecte ni genere complicaciones en el estudiante, es importante tener presente que el ser humano cambia sus modos de pensar constantemente por lo que hace que interiormente el mismo hace que su pensamiento sea complejo puesto a que estamos expuestos a distintas situaciones cotidianas que provocan una serie de reacciones que obligan a nuestra mente a trabajar de forma causal dependiendo del momento por el que estemos pasando.

\section{LA NUEVA EDUCACION}

Según Rivarola (2000), Jarauto (2012), Larrea (1960), Tejedor (1996) y Benetti (1970) nos dicen de la nueva educación: El camino a la nueva educación exigirá muchos años de serio trabajo cooperativo y se considera importante cambiar el ahora y poner todo el empeño de los involucrados, lo certero de sus afirmaciones y la forma ágil y elegante en que esta expresado. Además, debe estar implementada la tecnología que está en constante innovación, elaborando nuestra propia visión del futuro y del presente, siendo el objetivo principal para el futuro de la educación llegando a "la nueva educación eficaz y versátil."

La educación se ha convertido en una cuestión de importancia fundamental en todo el mundo. Las necesidades de la educación bajo las condiciones modernas de las relaciones internacionales y a la luz de una nueva civilización emergente, no sólo ocupan la atención de esos países que ahora, por primera vez en la historia, proporcionan educación a las masas de sus pueblos, sino también la de países en los que hace ya tiempo que existe algún tipo de educación obligatoria.

Se puede decir que la educación es un tema a tratar muy enriquecido en enseñanza ya que todos buscan los métodos para alcanzar la clara compresión de teorías que nos ayuden a comprender mejor el entorno que nos rodea y de la misma forma nos facilite la vida.

Iniciaremos con Rivarola (2000) quien pone énfasis en el empeño de cada individuo. Debemos mencionar la importancia del llamado "empeño" y es que esta acción es fundamental para llevar a cabo cualquier objetivo que nos propongamos en la actualidad o en futuro. No podemos dejar a un lado por tanto el empeño ya que gracias a este podremos alcanzar una Educación de calidad, eficaz y versátil que nos ayude a ser más doctos en los diferentes temas ya sean en Administración, Docencia u temas referentes a la Informática , entre otros. 
Una vez claro la importancia del empeño del individuo considera valorar la importancia de los métodos de aprendizaje siendo unos más efectivos que otros pero además no podemos menospreciar a ninguno ya que para cada persona será un método más eficaz que el otro y esto definitivamente nos abre más claro el termino evolución de la llamada nueva educación.

Otro de los autores llamado Jarauto (2012) nos dice que la educación está en constante evolución; frente a esto mencionare la importancia por estar en continua actualización frente a las nuevas teorías, hipótesis, conjetura, probabilidad, especulación, para alcanzar a tener la respectiva educación con todas las actualizaciones pertinentes.

Además, los involucrados son muy importantes, refiriéndonos a los docentes y estudiantes. Para cualquier docente que ama su trabajo, para cualquier docente que se levanta todos los días con la firme convicción de que otra educación, de que una Nueva Educación es posible. Esta Nueva Educación es la escuela que parte del respeto, la cooperación, el amor a la profesión, la escucha activa y que convierte a tus alumnos, a mis alumnos, en los verdaderos protagonistas del cambio.

\section{PENSAMIENTO COMPLEJO EN LA EDUCACIÓN SUPERIOR}

La educación superior contiene consideraciones culturales y humanas que sustentan la esencia el desarrollo universitario, incuestionablemente vinculada a la intencionalidad formativa que constituye el propósito ineludible de la universidad, pero en relación dialéctica con la sistematización de la gestión formativa, desde de un punto de vista del resultado acumulado en la construcción del conocimiento por la humanidad y el desarrollo constante del conocimiento científico. En términos generales, el pensamiento complejo en la educación superior tiene que ver con la capacidad para razonar eficientemente.

Según (TOBON, 2008) "En el campo de la Didáctica de la Educación Superior, cuando se expresa cómo se desarrolla la sistematización del proceso formativo en el desarrollo del proceso de enseñanza y aprendizaje, lo que constituye el proceso educativo formativo, en que la Pedagogía de la Educación Superior aporta la interpretación y explicación de los procesos y fenómenos, que son empáticos a la formación profesional y social, por lo que establece la orientación teórico metodológica hacia la Didáctica en la Educación Superior"

(T, 2006) "Si el desarrollo del proceso de la educación superior a una dimensión de un alto nivel en la formación, es decir la formación profesional se desarrolla a través de la investigación de avanzada con un carácter profesionalizante, que se sustenta en la Pedagogía. Luego entonces esta Didáctica tiene como objeto el desarrollo del proceso de enseñanza-aprendizaje en la formación de profesionales que es un proceso social intencional específico. Esto lleva a una reflexión muy interesante que se direcciona hacia el sustento del hecho de que la Didáctica es una ciencia inherente a la Pedagogía que tiene una especificidad respecto a la Pedagogía, pero que en esa especificidad hay una riqueza y diversidad como interpretación de lo holístico y complejo del proceso formativo “

El desarrollo del proceso de enseñanza-aprendizaje en la formación de profesionales, es un proceso social intencional específico, se sustenta en el movimiento dialéctico de unidad y 
contradicción entre la orientación sistematizadora y la generalización formativa, no como movimiento lineal de uno al otro, sino como proceso dialéctico.

Según (DURAN, 2006) "Al asumir los niveles de continuidad y gradualidad del proceso de formación de los profesionales desde la relación entre la generalización formativa y el proceso pedagógico ofrece un cuadro exhaustivo de la comprensión científica de los mecanismos y medios pedagógicos posibles de conexión en su carácter ascensional en la formación de los profesionales, que se realiza con una intencionalidad y una sistematización, y debe entenderse como un proceso coherente, en progreso y cíclicamente, que reafirma y crea a la cultura del contexto y la cultura universal."

La Educación Superior. Significa el desarrollo del proceso de enseñanza-aprendizaje a una dimensión de un alto nivel en la formación, es decir la formación profesional se desarrolla a través de la investigación de avanzada con un carácter profesionalizante.

(I, 1998) "La Educación Superior se erige a partir de la orientación sistematizadora en el proceso como tal, la cual propicia las cualidades de enseñabilidad y aprendibilidad, en un proceso único de enseñanza-aprendizaje. No como procesos independientes, sino como cualidades que se alcanzan en unidad."

Por consiguiente, las universidades requieren ofrecer respuestas a la formación de los profesionales para el mundo complejo que se desarrolla vertiginosamente, por demás, con asimetrías económicas y sociales, que implica la necesidad no sólo de la formación, sino de las investigaciones y la vinculación social, para que llegue a ser un espacio permanente de desarrollo cultural que incorpore a todos y durante toda la vida.

Pensamiento complejo: muestra las capacidades de intercomplementar varias dimensiones de lo real ante emergencias de objetos interactivos y competentes aleatorios, el involucrado con ello tiene que desarrollar estrategias de pensamiento las cuales deben ser reflexivas. Se denomina a dicha capacidad como pensamiento complejo

El pensamiento complejo se opone a la división disciplinaria y promueve un enfoque global, aunque sin abandonar la noción de las partes que constituyen un todo. La sistémica, la cibernética y las teorías de la información aportan sustento al pensamiento complejo. Puede decirse que el pensamiento complejo se basa en tres principios fundamentales: la dialogía (la coherencia del sistema aparece con la paradoja), la recursividad (la capacidad de la retroacción de modificar el sistema) y la hologramía (la parte en el todo y el todo en la parte).

El pensamiento complejo, por lo tanto, es una estrategia o forma del pensamiento que tiene una intención que globaliza los fenómenos pero que, a la vez, reconoce la especificidad de las partes. La clave pasa por la rearticulación de los conocimientos a través de la aplicación de los principios mencionados. Todo lo relacionado con el pensamiento complejo está vinculado a la epistemología (la doctrina de los métodos del conocimiento científico). El objeto de estudio de la epistemología es la producción y validación del conocimiento científico a través del análisis de distintos criterios. 
El término complejo suele ser asociado a aquellas cosas muy difíciles de comprender, inalcanzables para quienes no posean una serie de terminada de destrezas o conocimientos muy específicos. Sin embargo, su etimología nos muestra un significado ligeramente diferente: "lo que está tejido en conjunto". De allí que el pensamiento complejo, se base en establecer relaciones y complementos, en el estudio del todo mediante sus defectos y sus efectos, su movimiento y su quietud, tomando en cuenta la reciprocidad que tiene lugar entre éste y sus partes.

La complejidad representa al mundo como una gran red formada de delgados hilos que se entrelazan y relacionan todos sus componentes. El pensamiento complejo atiende cuestiones tan profundas pero tan cercanas como la vida, los problemas sociales y el futuro de la especie humana, en los últimos tiempos ha cobrado mucha importancia en el ámbito de la educación, el foco de las relaciones interpersonales más significativo, dado que representa el proceso de formación que nos vuelve aptos para integrarnos en la sociedad de una forma activa y productiva.

Todo pensamiento considerado complejo debe presentar una organización basada en la coherencia, estar formado de conceptos ricos y generar un constante movimiento, una necesidad de investigar y explorar. Asimismo, en más de una ocasión la importancia de inculcar a los estudiantes este tipo de pensamiento desde la infancia, para estimular su intelecto, su sentido crítico y su creatividad.

Se consideraba que era fundamental incluir la filosofía en el programa de toda escuela y colegio, para enriquecer los recursos educativos, ampliar el rango y la naturaleza de los conceptos que se enseñan e incentivar la autocorrección, la revisión de los propios métodos y del contenido que se imparte. Nuevamente, subrayaba la importancia de la crítica y de la creatividad como pilares de la educación.

Promover la reflexión como elemento fundamental del crecimiento parece una idea revolucionaria, casi rozando la ilusión, en un mundo gobernado por tendencias industriales; nos educan para encajar en uno $\mathrm{u}$ otro molde, para convertirnos en un modelo predeterminado de ser humano que transite por uno de un puñado de caminos posibles.

\section{PARADIGMAS EDUCATIVOS}

Según los autores: Juan Domingo, Martín Romero, Jorge Aranda, Flor Delgado y Luis Sarango. "El término Paradigma proviene del Griego "Paradigma" que significa modelo, patrón. Son conocimientos y creencias que forman una visión del mundo como modelo de una situación dada lo cual miramos al mundo desde un determinado marco, lo comprendemos, lo interpretamos e intervenimos sobre él, abarca desde el conjunto de conocimientos científicos en un sentido amplio el Paradigma corresponde como modelo o ejemplo a seguir en una situación dada, en torno a una teoría hegemónica está constituido por supuestos teóricos, leyes y técnicas de aplicación que deberán adoptar las personas."

Las personas a través de los tiempos han ido cambiado, al igual que la educación que es una actividad del quehacer humano y ha evolucionado de acuerdo a las condiciones de la 
humanidad y a aquellos pensadores y los luchadores incansables que no conformes, buscan el renovar el hacer educativo que permita una mejor forma de aprender y de enseñar. Esta es la razón por la cual en la educación es necesario retomar los aciertos de cada una de las formas que han existido en el proceso enseñanza aprendizaje. Estas formas son llamadas Paradigmas y su estudio permitirá implementar la mejor estrategia para evolucionar a nuevas formas y herramientas para aprender a conocer mejor al mundo y lo necesario para saber entender lo científico de la vida.

En este caso hay que tomar en cuenta la educación antigua que vendría hacer los paradigmas y saber que la educación actual está en una nueva ciudad del conocimiento ya sea con ayuda de la tecnología hoy en día se ha implementado las NTICS ( Nuevas Tecnologías de la Información y Comunicación) que no solo son de gran ayuda en la educación sino en varios ámbitos.

Sabiendo así que el término Paradigma proviene del Griego Paradigma, que significa Modelo, Patrón, se puede definir entonces como el resultado de los usos y costumbres, de creencias establecidas de verdades a medias. Técnicamente los paradigmas son un conjunto de conocimientos y creencias que forman una visión del mundo, en torno a una teoría hegemónica en determinado periodo histórico. Cada paradigma se instaura tras una revolución científica, que aporta respuestas a los enigmas que no podían resolverse en el paradigma anterior.

Los paradigmas cumplen una doble función, por un lado, la positiva que consiste en determinar las direcciones en las que ha de desarrollarse la ciencia normal, por medio de la propuesta de enigmas a resolver dentro del contexto de las teorías aceptadas. Por otro lado la función negativa del paradigma, es la de establecer los límites de lo que ha de considerarse ciencia durante el tiempo de su hegemonía.

El paradigma, está constituido por supuestos teóricos, leyes y técnicas de aplicación que deberán adoptar las personas que se mueven dentro de una determinada comunidad científica. Los que trabajan dentro de un paradigma, ponen en práctica la ciencia normal. Es probable que al trabajar en ella, en su intento por explicar el comportamiento de aspectos del mundo, se encuentren dificultades. Si estas dificultades se hacen inmanejables, se desarrollará un estado de crisis. Ésta se resolverá con el surgimiento de un paradigma completamente nuevo, el cual cobrará cada vez mayor adhesión o aceptación por parte de la comunidad científica, hasta que finalmente se abandone el paradigma original. Este cambio no es continuo, sino por el contrario es discontinuo y constituye una revolución científica. El nuevo paradigma enmarcará la nueva actividad científica normal, hasta que choque con dificultades y se produzca una nueva crisis y una nueva revolución y por lo tanto el surgimiento de un nuevo paradigma.

Los líderes científicos con sus corrientes psicológicas, son los que han dejado una enorme huella en educación y presentan los cinco paradigmas psicopedagógicos: Conductista, Cognitivo, Histórico-Social y Constructivismo que han sido considerados como modelos a seguir en el proceso enseñanza aprendizaje. 
El Paradigma conductista enfoca que el aprendizaje debe enfocarse en fenómenos observables y medibles. Sus fundamentos nos hablan de un aprendizaje producto de una relación "estímulo - respuesta". Los procesos internos tales como el pensamiento y la motivación, no pueden ser observados ni medidos directamente por lo que no son relevantes a la investigación científica del aprendizaje. El aprendizaje únicamente ocurre cuando se observa un cambio en el comportamiento. Si no hay cambio observable no hay aprendizaje.

La teoría Cognitiva proporciona grandes aportaciones al estudio de los procesos de enseñanza y aprendizaje, como la contribución al conocimiento preciso de algunas capacidades esenciales para el aprendizaje, tales como: la atención, la memoria y el razonamiento. Muestra una nueva visión del ser humano, al considerarlo como un organismo que realiza una actividad basada fundamentalmente en el procesamiento de la información, muy diferente a la visión reactiva y simplista que hasta entonces había defendido y divulgado el conductismo. Reconoce la importancia de cómo las personas organizan, filtran, codifican, categorizan, y evalúan la información y la forma en que estas herramientas, estructuras o esquemas mentales son empleadas para acceder e interpretar la realidad.

El Paradigma histórico social, también llamado paradigma sociocultural o histórico cultural, fue desarrollado por Vigotsky a partir de la década de 1920. Este paradigma establece que el individuo, aunque importante, no es la única variable en el aprendizaje. Su historia personal, su clase social y consecuentemente sus oportunidades sociales, su época histórica, las herramientas que tenga a su disposición, son variables que no solo apoyan el aprendizaje sino que son parte integral de él. Estas ideas lo diferencia de otros paradigmas.

Una premisa central de este paradigma es que el proceso de desarrollo cognitivo individual no es independiente o autónomo de los procesos socioculturales en general, ni de los procesos educacionales en particular. No es posible estudiar ningún proceso de desarrollo psicológico sin tomar en cuenta el contexto histórico-cultural en el que se encuentra inmerso, el cual trae consigo una serie de instrumentos y prácticas sociales históricamente determinados y organizados.

El Paradigma Constructivista sostiene que el aprendizaje es esencialmente activo. Una persona que aprende algo nuevo, lo incorpora a sus experiencias previas y a sus propias estructuras mentales. Cada nueva información es asimilada y depositada en una red de conocimientos y experiencias que existen previamente en el sujeto, como resultado podemos decir que el aprendizaje no es ni pasivo ni objetivo, por el contrario es un proceso subjetivo que cada persona va modificando constantemente a la luz de sus experiencias.

El constructivismo busca ayudar a los estudiantes a internalizar, reacomodar, o transformar la información nueva. Esta transformación ocurre a través de la creación de nuevos aprendizajes y esto resulta del surgimiento de nuevas estructuras cognitivas que permiten enfrentarse a situaciones iguales o parecidas en la realidad. 
El estudio de los paradigmas educativos es lo que prepara principalmente al estudiante para entrar en forma como miembro de la comunidad científica particular con la que trabajara más tarde.

\section{LA SOCIEDAD DEL CONOCIMIENTO}

(López, 2011) "La sociedad del conocimiento está involucrada en las actividades económicas, sociales, y culturales. También es conocida como el recurso principal que se crea, comparte y utiliza para la prosperidad de sus miembros. Este concepto, es el factor de la producción que dirige a la economía y desarrollo social. Por ello es comprensible, la evolución de la humanidad a través de los avances en el ámbito. Por lo tanto es importante tener en cuenta que el contexto mundial es cambiante, indicando que este concepto es refutable."

El término 'sociedad del conocimiento' ocupa un lugar estelar en la discusión actual en las ciencias sociales así como en la política europea. Se trata de un concepto que aparentemente resume las transformaciones sociales que se están produciendo en la sociedad moderna y sirve para el análisis de estas transformaciones. Al mismo tiempo, ofrece una visión del futuro para guiar normativamente las acciones políticas

El conocimiento teórico se ha convertido, según este enfoque, en la fuente principal de innovación y el punto de partida de los programas políticos y sociales. Este tipo de sociedad está orientado hacia el progreso tecnológico y la evaluación de la tecnología y se caracteriza por la creación de una nueva tecnología intelectual como base de los procesos de decisión.

La noción sociedad de conocimiento tiene sus orígenes en los años 1960 cuando se analizaron los cambios en las sociedades industriales y se acuñó la noción de la sociedad post-industrial. Así, por ejemplo, el sociólogo Peter F. Drucker pronosticó la emergencia de una nueva capa social de trabajadores de conocimiento (P.F. Drucker 1959) y la tendencia hacia una sociedad de conocimiento (Drucker 1969). Este tipo de sociedad está caracterizada por una estructura económica y social, en la que el conocimiento ha substituido al trabajo, a las materias primas y al capital como fuente más importante de la productividad, crecimiento y desigualdades sociales (Drucker 1994).

Estos análisis apuntaron, por lo tanto, a una sociedad cientificada, academizada y centrada en los servicios diferenciada de la sociedad industrial que, a su vez, estaba caracterizada por el conocimiento experimental, el predominio de los sectores industriales, las actividades manuales y el conflicto entre capital y trabajo.

Desde luego, el concepto de la 'sociedad del conocimiento' no es el único empleado para describir los cambios sociales en las sociedades actuales. También se usan otras nociones como, por ejemplo, la 'sociedad de la información' y la 'sociedad red'. (CONDE, 2010 ) "Las sociedades de la información emergen de la implantación de las tecnologías de información y comunicación (TIC) en la cotidianidad de las relaciones sociales, culturales y económicas en el seno de una comunidad, y de forma más amplia, eliminando las barreras 
del espacio y el tiempo en ellas, facilitando una comunicación ubicua y asíncrona. La eficacia de estas nuevas tecnologías -actuando sobre elementos básicos de la persona como el habla, el recuerdo o el aprendizaje-, modifica en muchos sentidos la forma en la que es posible desarrollar muchas actividades propias de la sociedad moderna."

La noción de la 'sociedad de la información' se utiliza sobre todo cuando se tratan aspectos tecnológicos y sus efectos sobre el crecimiento económico y el empleo, Esta discusión tiene como punto de partida la consideración de que la producción, la reproducción y la distribución de la información es el principio constitutivo de las sociedades actuales. Pero en la reciente discusión europea, tanto en el ámbito de las ciencias sociales como en el ámbito político, se observa que este término es reemplazado por el de la 'sociedad del conocimiento lo que implica un cambio conceptual de la información al conocimiento considerándolo como principio estructurador de la sociedad moderna y resaltando su importancia para la sociedad actual, para los cambios en la estructura económica y en los mercados laborales, para la educación y para la formación.

Similar al concepto de la 'sociedad del conocimiento', el de la 'sociedad red' sostiene que la transformación actual de la sociedad indica un cambio de modo de producción social, dada la creciente importancia de la información o del conocimiento para los procesos socioeconómicos. Información y conocimiento se convierten en los factores productivos más importantes. Sin embargo, y a pesar de que Castells declara que ni la tecnología determina la sociedad ni la sociedad dicta el curso del cambio tecnológico, el enfoque de la sociedad red parte de la idea de una tecnología hecha e investiga los efectos de la aplicación de los inventos tecnológicos sobre la sociedad. No tiene en cuenta que la tecnología es una construcción social en cuyo desarrollo intervienen múltiples actores y diferentes intereses y tampoco profundiza más en la idiosincrasia del conocimiento y su relevancia para los procesos socio-económicos y los efectos sobre el propio conocimiento cuando se ha convertido en el factor productivo más importante.

Desde los años 1990, el concepto de la 'sociedad del conocimiento' ha resurgido tanto en la política como en las ciencias sociales. Al contrario que el concepto de la 'sociedad de la información', este término no hace referencia solamente a la base tecnológica para caracterizar la sociedad actual y para resaltar las diferencias con la 'sociedad industrial'.

\section{CONCLUSIONES}

1. Similar al término 'sociedad de la información', la noción 'sociedad de conocimiento' indica la importancia de las tecnologías de la información y la comunicación (TIC) y su utilización en los procesos económicos.

2. La noción resalta las nuevas formas de producir conocimiento. El conocimiento es considerado como uno de los principales causantes del crecimiento junto con los factores capital y trabajo. En este sentido, se concede una relevancia crucial a la producción de productos intensivos en conocimiento y a los servicios basados en el conocimiento

3. Se resalta la creciente importancia de los procesos educativos y formativos, tanto en su vertiente de educación y formación inicial como a lo largo de la vida. 
4. Se destaca la creciente importancia de los servicios intensivos en conocimiento y comunicación, que generalmente se denominan trabajo de conocimiento

5. El concepto de "sociedad del conocimiento" hace referencia, por lo tanto, a cambios en las áreas tecnológicas y económicas estrechamente relacionadas con las TIC, en el ámbito de planificación de la educación y formación, en el ámbito de la organización (gestión de conocimiento) y del trabajo (trabajo de conocimiento).

RECEIVED: SEPTEMBER, 2016 REVISED: DECEMBER, 2016

\section{REFERENCIAS BIBLIOGRAFICAS}

1. Beatriz Jarauta, F. I. (2012). Pensando en el futuro de la educación. Barcelona: Editorial Grao.

2. Benetti, S. (1970). Una educación nueva para un hombre nuevo. Roma: Editorial Bonum.

3. Bilancio, G. (2006). Estrategia: equilibrio entre el caos y el orden para anticipar. Chile: Pearson Educación.

4. Caballero, L. (2008). La búsqueda del Comienzo: El pensamiento complejo . México : CopIt ArXives Editoriales.

5. Castillo, D. P. (1998). La comunicación en la educación. Cuyo.

6. Costa, J. L. (2008). el desarrollo de la competencia experta: implicaciones para la enseñanza. Club Universitario: San Vicente (Alicante).

7. Duran, M. (2006). Universitario ante el espacio europeo de la educación superior . Académicos .IEMS.

8. González, C. H. (2012). Conocimiento de la vida. E.E.U.U: Palibrio.

9. Grinberg, M. (2002). Edgar Morín y el pensamiento complejo. España: Campo de Ideas Editorial.

10. I, A. (1998). Problemas lógicos de la educación superior . Madrid: progreso.

11. Ivan, A. (1999). El procesó y su movimiento en la educación superior. Madrid: MC. GRAN WILL.

12. Jiménez, E. S. (s.f). La Hojarasca Alianza de escritores y periodistas. Obtenido de http://www.escritoresyperiodistas.com/Numero31/Numero31.htm

13. Kaplúm, M. (2003). Procesos educativos y canales de comunicación. Obtenido de http://www.eca.usp.br/nucleos/nce/pdf/115.pdf

14. Laclette, J. P., Zuñiga, P., \& Puga Espinoza, C. (2010). Informe mundial sobre las ciencias sociales: las brechas del conocimiento. México D.F.: Unesco.

15. Larrea, J. C. (1960). La educación nueva: significados, fines, organización, métodos, la formación del maestro, la educación para la comprensión internacional. Ecuador: Casa de la cultura Ecuatoriana.

16. Lipman, M. (2014). Pensamiento complejo y educación. España: Editorial de la Torre.

17. Najmanovich, D. (2008). Mirar Con Nuevos Ojos Nuevos Paradigmas en la Ciencia y Pensamiento Complejo. Buenos Aires: Editorial Biblos. 
18. Olivos, M. A. (03 de marzo de 2005). El blog de Manuel Antonio Alva Olivos. Obtenido de http://manuelalvaolivos.obolog.es/importancia-comunicacioneducacion-60698

19. Rivarola, D. M. (2000). Nueva educación y democracia: realidad o utopía. Ediciones y Arte.

20. Rubio Matilla, V. (2011). Estrategia universidad 2015. Contribución de las universidades al progreso socio-económico. España: educacion.es.

21. Solana, J. (2005). Con Edgar Morín, por un pensamiento complejo. Madrid: Editorial Akal.

22. T, S. (2006). Calidad educación. Bogotá: alma mater.

23. Tejedor, F. J. (1996). Perspectivas de las nuevas tecnologías en la educación. Madrid: Narcea s.a. De ediciones.

24. Tiscar. (14 de mayo de 2006). Tiscar. Obtenido de comunicación y educación: http://tiscar.com/2006/05/14/comunicacion-y-educacion/

25. Tubon, S. (2008). Competencias a la educación superior. Regioncadeyta.org.

26. Romero, M. (2010). Paradigmas, Enfoques y Teorías Educativas: Histecalador

27. Delgado, F. (2012). Paradigmas y Retos de la Investigación Educativa. Universidad de los andes: Consejo de Publicaciones.

28. Aranda, J. (2009).Los Paradigmas de la Investigación y los enfoques. Perú.

29. Sarango, L. (2010). Paradigma educativo de Abya Ayala. Nicaragua.

30. Casadevall, I. T. (2011). Sociedad del conocimiento. España: copyright.

31. Conde, J. R. (2010). Investigación y evolución educativas en la sociedad del conocimiento. España: salamanca.

32. Duran, C. R. (2011). El reto de la educación superior en la sociedad del conocimiento. México: biblioteca de la educación superior.

33. López, F. M. (2011). Marketing y la sociedad del conocimiento. Madrid: delta.

34. Rojas, J. M. (2010). Gestión educativa de la sociedad del conocimiento. Colombia: magisterio.

35. Domingo, J. (2010). Recuperado de: https://www.academia.edu/3224671/Paradigmas_Educativos

\section{【L Ciencia}

El artículo que se publica es de exclusiva responsabilidad de los autores y no necesariamente reflejan el pensamiento de la Revista Ciencia Digital.

El articulo queda en propiedad de la revista y, por tanto, su publicación parcial y/o total en otro medio tiene que ser autorizado por el director de la Revista Ciencia Digital.
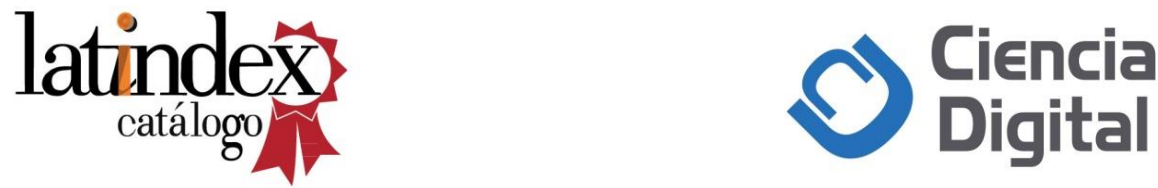